\title{
Modulating the cardiotoxic behaviour of immunoglobulin light chain dimers through point mutations
}

Martina Maritan ${ }^{1}$, Arianna Ambrosetti ${ }^{1}$, Luca Oberti ${ }^{1}$, Alberto Barbiroli ${ }^{2}$, Luisa Diomede ${ }^{3}$, Margherita Romeo ${ }^{3}$, Francesca Lavatelli ${ }^{4}$, Pietro Sormanni ${ }^{5}$, Giovanni Palladini ${ }^{4}$, Martino Bolognesi ${ }^{1}$, Giampaolo Merlini ${ }^{4}$ and Stefano Ricagno .

${ }^{1}$ Department of Bioscience, University of Milan, Milan, Italy;

${ }^{2}$ Department of Nutritional Science, University of Milan, Milan, Italy;

${ }^{3}$ IRCCS-Istituto di Ricerche Farmacologiche "Mario Negri", Milano, Italy;

${ }^{4}$ Amyloidosis Research and Treatment Center, Policlinico San Matteo, Italy;

${ }^{5}$ Department of Chemistry, University of Cambridge, Cambridge, UK

Address for correspondence: Stefano Ricagno, Department of Biosciences, University of Milan, 20133 Milano, Italy. E-mail: stefano.ricagno@unimi.it

Background. Light chain amyloidosis (AL) is caused by the overproduction, misfolding and aggregation of immunoglobulin light chains (LCs) that tend to deposit as amyloid fibrils in the cardiac tissue ${ }^{1}$. Nowadays, the molecular details underling LCs soluble cardiotoxicity and fibril formation remain to be fully elucidated. It has been suggested a relationship between conformational flexibility and amyloidogenicity, indicating protein thermal stability and dynamics as factors able to influence the complex processes of misfolding and aggregation $^{2,3}$. To date, no compelling correlations have been identified between LCs cardiotoxicity and primary sequence. Here we present a pivotal mutagenesis study in which we produce and characterise a mutated variant of the toxic H6 LC (mH6). Our purpose is to abrogate the toxicity typical of H6 yielding to a non-cardiotoxic LC and to understand the biophysical and biochemical changes underlying such loss of toxicity.

Material and Methods. H6 mutant design was carried out combining the results of bioinformatics tools for protein stability predictions and sequence multi-alignment. H6 sequence was mutated into $\mathrm{mH} 6$ using side directed mutagenesis. E. coli was used to express LCs, which are then refolded and purified by chromatographic methods as previously reported ${ }^{3}$. For protein characterization both spectroscopic (Circular Dichroism, Fluorescence) and structural (X-ray crystallography) techniques were applied. In-vivo toxicity assessment was carried out measuring C. elegans' pharynx pumping rate ${ }^{4}$.

Results. The design of mutated H6 was performed applying an innovative method that correlates structural data with toxicity (Figure 1). A set of thermal stabilizing mutation candidates were found through an hybrid approach involving: i) multi-sequence analysis and ii) energetic calculations. H6 amino acid sequence was aligned against a multiple myeloma sequence database, in order to identify the conserved amino acids spots associated with nontoxic LC (Figure 1A). The crystal structure of H6 was analysed by bioinformatics tools that calculated the most favourable mutations to achieve protein stabilisation (Figure 1B). Final mutations selection was obtained by merging and ranking the best outcomes of the two methods (Figure 1C), which led to the identification of three point mutations located in the variable domain.

Preliminary data on mH6 in vivo toxicity seem to indicate a significant decrease in the mutant toxic profile compared to the wild type cardiotoxic H6. This points out the critical role of specific amino acid positions in determining the toxic behaviour.

Interestingly, the melting temperatures of $\mathrm{H} 6$ and $\mathrm{mH} 6$ monitored by Far-UV circular dichroism are comparable $\left(\mathrm{T}_{\mathrm{m}} \mathrm{H} 6=43.9-54.5^{\circ} \mathrm{C}, \mathrm{T}_{\mathrm{m}} \mathrm{mH} 6=53.8^{\circ} \mathrm{C}\right)$. However unfolding profiles are not perfectly superposable suggesting different unfolding transitions: $\mathrm{mH} 6$ 
appear to have a more defined transition, which possibly reflect a more compact domain organization than $\mathrm{H} 6$ three step unfolding ${ }^{3}$. Limited proteolysis data revealed slower proteolytic kinetics for the mutant, hence reduced fold stability.

The crystal structure of $\mathrm{mH} 6$ has been determined at $2.1 \AA$ resolution, displaying a homodimeric organization common to all LC dimers. Secondary structure superposition does not show evident differences between the H6 structure and the $\mathrm{mH} 6$ one, giving an r.m.s.d. value of $0.3 \AA$ for the overall dimers superposition, indicating that the mutagenesis has no impact on the native protein organization and dimerization.

A

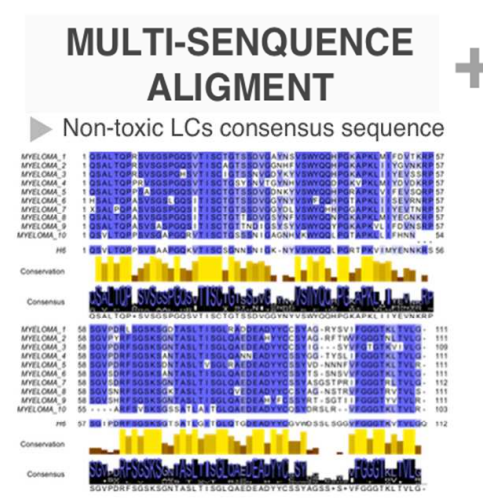

B

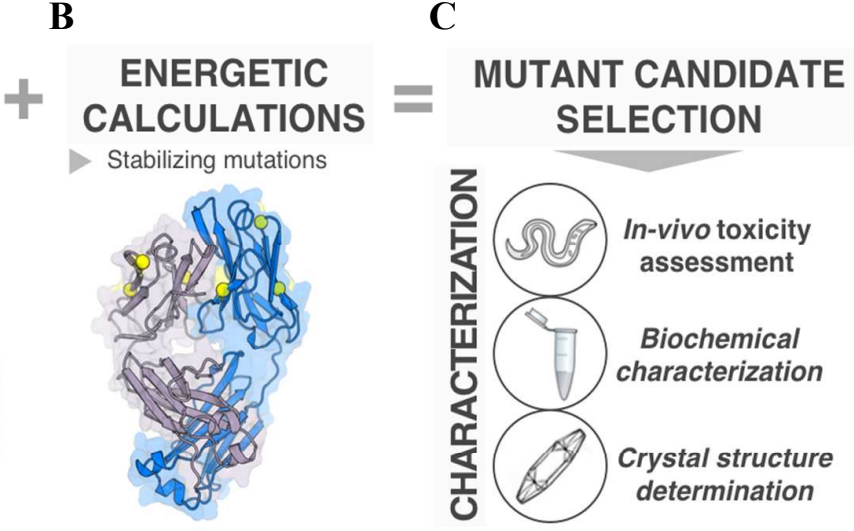

Figure 1. (A) Representative sequence multi-alignment of myeloma LCs against H6. Sequence conservation and consensus sequence are depicted by histograms. (B) Bioinformatics analysis using H6 structure as input run to identify possible mutation spots. (C) The combination of outputs from (A) and (B) lead the mutation spot selection and subsequent protein production and characterization.

Discussion and conclusions. Previous biophysical findings indicate that low fold stability and high protein dynamics correlate with LC amyloidogenicity ${ }^{3}$. Our data showed that the toxicity of a soluble LC could be tuned by introducing specific point mutations. This evidence points out the importance of the primary sequence for cardiotoxic LCs. Moreover, our data strengthen the hypothesis that links LCs toxic behaviour in vivo to thermal stability and dynamics.

Declaration of interest. The authors report no conflict of interest.

\section{References}

1 Ramirez-Alvarado, M. Amyloid formation in light chain amyloidosis. Current topics in medicinal chemistry. 2012; 2523-2533.

2 Poshusta, T. L., Katoh, N., Gertz, M. A., Dispenzieri, A. \& Ramirez-Alvarado, M. Thermal stability threshold for amyloid formation in light chain amyloidosis. International journal of molecular sciences. 2013; 22604-22617.

3 Oberti, L. et al. Concurrent structural and biophysical traits link with immunoglobulin light chains amyloid propensity. Scientific reports. 2017; 7.1.

4 Diomede, L. et al. A Caenorhabditis elegans-based assay recognizes immunoglobulin light chains causing heart amyloidosis. Blood. 2014; 123:3543-3552. 